\title{
IMPACTO DE LAS FUSIONES Y ADQUISICIONES EN LA EFICIENCIA DE LA BANCA EN COLOMBIA*
}

\author{
Miguel Sarmiento \\ Hernando Mutis ${ }^{\mathrm{b}}$ \\ Andrés Cepedac \\ Juan F. Pérez
}

*DOI: https://doi.org/10.18601/01245996.v20n38.07. Una versión anterior de este trabajo fue presentada por Andrés Cepeda como parte de su tesis de Magíster en Ingeniería Industrial de la Universidad de los Andes. Las opiniones aquí expresadas son de exclusiva responsabilidad de los autores, así como los posibles errores u omisiones. Los autores agradecen los comentarios de dos evaluadores anónimos y las sugerencias de Clara Machado, Sandra Benítez y Orlando Chipatecua. Recepción: 30-08-2016, modificación final: 21-01-2018, aceptación: 19-02-2018. Sugerencia de citación: Sarmiento, M., Mutis, H., Cepeda, A. y Pérez, J. F. (2018). Impacto de las fusiones y adquisiciones en la eficiencia de la banca en Colombia, Revista de Economía Institucional 20(38), 157-183.

a Candidato a doctor en Economía, economista del Banco de la República y profesor de la Universidad Externado de Colombia, Bogotá, Colombia, [nsarmipa@banrep.gov.co].

b Doctor en Economía, profesor de la Universidad de los Andes, Bogotá, Colombia,[hemutis@uniandes.edu.co].

c Magíster en Ingeniería Industrial, consultor de Mobility, Madrid, España [ha.cepeda54@uniandes.edu.co].

d Doctor en Ciencias de la Computación, profesor de la Universidad del Rosario, Bogotá, Colombia, [juanferna@urosario.edu.co]. 


\section{Impacto de las fusiones y adquisiciones en la eficiencia de la banca en Colombia}

Resumen. En este artículo se evalúa la eficiencia de la banca colombiana utilizando modelos de frontera no paramétricos. Los resultados indican que la industria bancaria ha presentado una tendencia creciente en su eficiencia, afectada por la crisis financiera global de 2008. A partir de 2009 se observa una rápida recuperación en las medidas de eficiencia, a excepción de la eficiencia en costos, lo cual sugiere que, ante choques externos, los costos de ajuste de la industria bancaria son mayores. Las fusiones y adquisiciones dentro de la industria contribuyen de forma importante al incremento en la eficiencia bancaria.

Palabras clave: frontera de eficiencia, sistema financiero, métodos no paramétricos, fusiones y adquisiciones; JEL: C14, G34, D24

\section{Impact of mergers and acquisitions on the efficiency of banking in Colombia}

Abstract. This article evaluates the efficiency of Colombian banking using nonparametric border models. The results indicate that there has been a gradual increase in efficiency and that the global financial crisis of 2008 affected this trend. Since 2009 there has been a rapid recovery of efficiency measures, except for cost efficiency. This suggests that, in the face of external shocks, the adjustment costs of banks are greater. Mergers and acquisitions contribute to the increase in banking efficiency.

Keywords: efficiency frontier, financial system, non-parametric methods, fusions and acquisitions; JEL: C14, G34, D24

\section{Impacto das fusões e aquisições na eficiência do setor bancário na Colômbia}

Resumo. Este artigo avalia a eficiência do setor bancário colombiano utilizando modelos de fronteira não paramétricos. Os resultados indicam que ocorreu um aumento gradual da eficiência e que a crise financeira global de 2008 afetou esta tendência. Desde 2009, observa-se uma rápida recuperação das medidas de eficiência, com a exceção da eficiência sobre custos; isto sugere que, diante de choques externos, os custos de ajuste do setor bancário são maiores. As fusões e aquisições contribuem para o aumento da eficiência bancária.

Palavras-chaves: fronteira de eficiência, sistema financeiro, métodos não paramétricos, fusões e aquisições; JEL: C14, G34, D24 
$\mathrm{U}$ n manejo eficiente de los recursos empleados en la operación de la banca se traduce en menores costos de intermediación financiera, lo cual estimula el crecimiento económico y contribuye al desarrollo (Dolar y Meh, 2002; Claus et al., 2004). La eficiencia bancaria se alcanza mediante diferentes mecanismos, entre estos, las fusiones y las adquisiciones (F\&A) han ganado especial relevancia. Si bien las F\&A buscan el incremento del poder de mercado, en este proceso las firmas más eficientes (nacionales o extranjeras) toman posesión de las menos eficientes, con el fin de aprovechar economías de escala y de alcance, lo cual se traduce en ganancias en eficiencia para todo el sistema financiero (Carletti et al., 2007; Koetter, 2008; Davis, 2007; DeYoung et al., 2009).

Entre 2000 y 2009 se realizaron 21 procesos de F\&A en la industria bancaria de Colombia, siendo la década con mayor cantidad de reorganizaciones en la banca. Por una parte, esta estrategia lleva a tener menos entidades de mayor tamaño en el sector, lo cual exige una regulación prudencial para evitar una concentración que limite la competencia y exacerbe el riesgo sistémico ${ }^{1}$. Por otra parte, si las reorganizaciones en la industria mejoran la eficiencia y reduce los costos de intermediación sería adecuado propiciar un ambiente favorable para las F\&A; que es precisamente el aporte de este artículo al brindar evidencia sobre el impacto de las F\&A en la eficiencia de la banca en Colombia.

Este artículo busca responder estas preguntas: ¿Cómo medir la eficiencia de una entidad financiera? ¿Cuál ha sido la evolución de la eficiencia de las entidades bancarias en Colombia? ¿Cuál ha sido el impacto de las F\&A sobre la eficiencia de las entidades absorbentes? Y hace tres contribuciones a la literatura colombiana: 1) a nivel metodológico, estima diferentes medidas de eficiencia de la industria bancaria utilizando el método no paramétrico de análisis envolvente de datos (DEA), que tiene ventajas sobre los métodos convencionales de frontera estocástica (Fiorentino et al., 2006; Staub et al., 2010); 2) a nivel empírico, evalúa el periodo 2000-2009 aportando nueva evidencia de una década de fuertes choques internos y externos (crisis financiera internacional) que afectaron el desempeño del sistema

${ }^{1}$ La concentración del sistema financiero ha propiciado la presencia de entidades sistémicamente importantes o Too-big-to-fail, lo cual exige una regulación macroprudencial con el fin de mitigar los efectos de contagio derivados de una falla de liquidez por parte de este tipo de entidades. Una evaluación del riesgo sistémico para el caso colombiano se puede consultar en León et al. (2011). Sobre el reciente efecto de las políticas de regulación macroprudenciales sobre la banca colombiana ver Gómez et al. (2016) y Dias et al. (2017). 
financiero; y 3) identifica el efecto de las F\&A en la eficiencia de las entidades absorbentes.

El artículo consta de cinco secciones: en la primera se discuten los estudios sobre medición de la eficiencia del sistema financiero, con especial énfasis en el caso colombiano. En la segunda se expone la metodología del análisis envolvente de datos (DEA) y los modelos empleados en este estudio. En la tercera se describen las variables y los datos. La cuarta presenta los resultados, en especial la evaluación del impacto de las F\&A sobre la eficiencia de las entidades absorbentes. La quinta presenta las conclusiones.

\section{MEDICIÓN DE LA EFICIENCIA}

El cálculo de las medidas de eficiencia se realiza a través de la comparación entre el punto de producción observado y un punto óptimo o teórico de producción que conforma la frontera de eficiencia. Cuando la frontera es calculada a partir de una función de producción, donde la cantidad de producto está en función de la cantidad de los insumos empleados se tiene la eficiencia técnica. En cambio, si la frontera es calculada con una función de costos, donde se relaciona el costo total de producción como función del nivel de producto, la cantidad de insumos y sus precios relativos, dicha frontera estará conformada por los procesos que exhiben eficiencia técnica y de asignación.

A partir de los conceptos de eficiencia de Farell (1957) se han desarrollado varias metodologías para medir la eficiencia relativa de las firmas empleando diferentes técnicas para su estimación, las cuales se agrupan en el enfoque paramétrico y el no paramétrico. La principal diferencia de estos métodos radica en la forma de construcción de la frontera de eficiencia y en la concepción acerca del término de ineficiencia y/o de la distribución asociada a este. El método paramétrico más aplicado es el Stochastic Frontier Analysis (SFA) propuesto por Aigner et al. (1977), el cual se basa en la estimación de una función de costos o de producción (típicamente Cobb Douglas o Trans-log) cuyos parámetros permiten caracterizar la frontera de eficiencia. Así, mediante el SFA se estima una frontera de producción o de costos donde los productos o los costos están en función de los insumos. La eficiencia es definida mediante una frontera a la cual se le incorpora un componente de ruido aleatorio que se descompone en dos partes: una puramente aleatoria que no está bajo el control de la firma y obedece a factores externos a ella; la otra parte se refiere a la ineficiencia. La inclusión del término aleatorio es una de las principales ventajas del SFA, pues reconoce que no toda ineficiencia detectada radica 
en la operación de las firmas, sino que también obedece a choques aleatorios a los que se ve sometidas las firmas. Sin embargo, una de las principales desventajas del SFA son los supuestos utilizados en la definición de la frontera estocástica, como lo son la forma funcional, por la cual se rige la conversión de los insumos en productos, y las distribuciones de ineficiencia y error (Coelli, Prasada y Battese, 1998).

Al no imponer una forma funcional específica para caracterizar el proceso de producción, el DEA no es susceptible de errores causados por la especificación de la tecnología de producción. Asimismo, este método permite considerar modelos con múltiples insumos y productos, además, debido a que la información con la cual se construye la frontera está dada por optimizaciones individuales de las firmas, se pueden aceptar comportamientos de tecnologías particulares para cada una de ellas (Cooper et al., 2004; Fiorentino et al., 2006; Staub et al., 2010). A su vez, es menos exigente con la muestra de datos empleada para la estimación de la frontera; mientras que los métodos paramétricos requieren una elevada cantidad de datos para lograr estimadores adecuados $^{2}$. Fiorentino et al. (2006) hacen un estudio comparativo de eficiencia bancaria en costos para los bancos alemanes, utilizando las aproximaciones DEA y SFA, y encuentran que los resultados de eficiencia en el análisis SFA están muy por encima de su contraparte en DEA. Evidencia que también fue encontrada por Berger y Mester (1997) en su extensa revisión de literatura.

\section{EFICIENCIA BANCARIA Y EVIDENCIA EN COLOMBIA}

En este estudio empleamos la metodología DEA para evaluar la eficiencia de la banca en Colombia. Este método no paramétrico ha sido utilizado en estudios internacionales para la medición de eficiencia en el sector bancario de Estados Unidos y Europa (Wheelock y Wilson, 1999; Altunbas et al., 2001; Alam, 2001; Weill, 2004). Un elemento importante en estos modelos es la definición adecuada del conjunto de insumos y productos, la cual se realiza mediante el enfoque de operación del banco. El enfoque de producción asume que los depósitos son productos del banco y no insumos, enfoque que ha sido revaluado en la literatura dado que los depósitos constituyen el principal insumo de un banco en su actividad de intermediación

${ }^{2}$ La flexibilidad del método DEA para medir eficiencia en sectores donde no existe una amplia disponibilidad de datos o en donde la cantidad de unidades a evaluar es muy reducida ha sido una de sus mayores ventajas frente al método de frontera estocástica. Por ello, su aplicación en evaluaciones de eficiencia para el sector público es más frecuente (Herrera y Pang, 2005; Sarmiento, 2006). 
financiera (Hughes y Mester, 1993). Desde entonces, la mayoría de los estudios emplean el enfoque de intermediación financiera donde los depósitos son uno de los principales insumos del modelo (Staub et al., 2010; Jemric y Vujcic, 2002).

La utilización de los modelos no paramétricos es relativamente reciente en Colombia. La primera aplicación para la banca se realizó en Berrío y Muñoz (2005) utilizando un modelo DEA para periodo 1993-2003. Sin embargo, los resultados obtenidos no fueron consistentes debido a la restricción excesiva del conjunto de posibilidades de producción. El trabajo de Almanza (2012) evalúa el periodo 1999-2007 con un modelo de dos etapas que calcula la eficiencia en costos bajo un modelo tipo DEA y luego en una segunda fase incluye un modelo Tobit para explicar los factores que afectan los índices de eficiencia estimados en la primera etapa. Este enfoque tiene el inconveniente de que si las variables de la segunda etapa resultan ser explicativas de las medidas de eficiencia, estas deberían ser incluidas en el modelo de frontera eficiente de la primera etapa, o de lo contrario los estimadores serían sesgados (Wang y Schmidt, 2002). Una alternativa es la estimación de un modelo SFA de una sola etapa donde las variables de entorno se incluyen como elementos que afectan la distribución de la ineficiencia (Sarmiento y Galán, 2017).El trabajo de Pirateque et al. (2013) emplea un modelo tipo DEA para evaluar la eficiencia de la banca en el periodo 2000-2012. Si bien los resultados son consistentes con los obtenidos en otros estudios para Colombia, emplea el enfoque revaluado de producción que toma los depósitos como productos del banco.

Este enfoque brinda evidencia sobre el impacto de las F\&A desde la óptica de los modelos no paramétricos, los cuales han ganado un espacio importante en la literatura por su flexibilidad, ya que no requieren el supuesto de una forma funcional específica que restringe el comportamiento de las entidades, así como supuestos adicionales sobre la distribución de la ineficiencia. Si bien se han realizado aplicaciones de estos modelos para Colombia, los estudios no han evaluado la influencia de las F\&A sobre la eficiencia, las cuales ocurrieron durante los periodos que analizan y que como veremos más adelante, tienen un impacto importante sobre los índices de eficiencia de los bancos. Adicionalmente, utilizan enfoques diferentes que afectan la selección de las variables, lo que puede conllevar a medidas de eficiencia inconsistentes. Esta desventaja es superada mediante el uso del enfoque de intermediación financiera que define claramente el conjunto de insumo y productos asociados a la producción bancaria. 
En cuanto al efecto de las F\&A sobre la eficiencia de la banca, se destaca el trabajo de Clavijo et al. (2006) quienes evaluaron las F\&A realizadas entre 1994 y 2005 empleando un modelo de frontera estocástica ${ }^{3}$. Los autores encuentran que las F\&A de mediados de los noventa se realizaron entre entidades pares con el fin de obtener poder de mercado y no con el fin de incrementar su eficiencia. Por su parte, los procesos realizados entre 2003 y 2005 fueron fusiones complementarias, que buscaban explotar economías de escala y alcance y, por ende, generaron ganancias en eficiencia. Galán et al. (2015) utilizan un modelo de frontera estocástica estimado bajo un enfoque Bayesiano para evaluar los procesos de F\&A entre 2000 y 2009. Los autores identifican que los bancos fusionados exhiben bajos costos de ajuste que les permiten recuperar rápidamente las pérdidas en eficiencia derivadas de los procesos de fusión (alrededor de dos años). Por esta razón, los resultados de Clavijo et al. (2006) sobre las fusiones realizadas entre 2003 y 2005 podrían ser débiles al no contemplar el tiempo necesario para la recuperación de los costos de las F\&A.

En este estudio empleamos el método DEA para evaluar el impacto de las F\&A sobre la eficiencia de la banca. La principal diferencia frente a los trabajos de Clavijo et al. (2006) y Galán et al. (2015) radica en la utilización del método de frontera eficiente, el cual presenta ventajas frente al SFA, tales como la no incorporación de una forma funcional específica para las firmas y la inclusión de la heterogeneidad, características esenciales de la industria bancaria. De esta forma, el estudio es pionero en la utilización de los modelos no paramétricos para evaluar el impacto de las F\&A sobre la eficiencia en la banca colombiana. Otra contribución del presente estudio, es el cálculo del cambio en productividad de Malmquist, el cual permite identificar si el incremento de la productividad obedece a un cambio técnico (que afectó a toda la industria bancaria) o a un acercamiento del banco a su frontera de eficiencia (ganancia en eficiencia). Como veremos más adelante, la capacidad de estimar varias medidas de eficiencia para un mismo grupo de entidades y el uso de análisis por ventanas de tiempo son otras ventajas de este método que tampoco han sido exploradas en los estudios previos.

${ }^{3}$ El desarrollo de los modelos de frontera estocástica ha permitido la incorporación de variables de entorno, capital financiero y riesgos entre otros aspectos que afectan la industria bancaria (Estrada y Osorio, 2004; Cepeda et al., 2010; Galán y Sarmiento, 2017). Sin embargo, estos estudios no evalúan el impacto de las F\&A sobre la eficiencia de la banca. 


\section{ENFOQUE METODOLÓGICO}

La metodología en este estudio parte del modelo no paramétrico DEA propuesto inicialmente por Charnes, Cooper y Rhodes (denominado CCR) y, posteriormente, se incorporan otros modelos bajo ciertos supuestos. El proceso para calcular las medidas de eficiencia con este enfoque se puede resumir en cuatro pasos: 1) seleccionar el conjunto de insumos y productos de las firmas o (Decision Making Units, DMU) y la orientación del modelo; 2) describir mediante ciertos supuestos las propiedades de la tecnología; 3) identificar el tipo de retornos a escala; 4) definir el tipo de índice de eficiencia y plantear un modelo (programa lineal) que permita el cálculo de los índices seleccionados.

Formalmente, en los modelo DEA se evalúan $n$ DMUs, donde cada una de ellas utiliza $m$ insumos distintos para producir $s$ productos diferentes. De esta manera, la DMU $j$ del análisis utiliza una cantidad $x_{i j} \geq 0$ del insumo $i$ para producir una cantidad $y_{r i} \geq 0$ del producto $r$. Así el modelo DEA CCR en su forma radial, usa la razón entre productos e insumos para medir la eficiencia relativa de la $D M U_{0}$ con respecto a las unidades $D M U_{j}$ para $j=1,2, \ldots, n$. El programa lineal (1) describe el modelo CCR, orientado a insumos, para la unidad $D M U_{0}$ :

$\theta^{*}=\min \theta$

$\sum_{j=1}^{n} x_{i j} \lambda_{j} \leq \theta x_{i 0}, i=1,2, \ldots, m$.

$\sum_{j=1}^{n} y_{r j} \lambda_{j} \geq y_{r 0}, r=1,2, \ldots, s$.

$\lambda_{j} \geq 0, j=1,2, \ldots, n$

El valor de $\theta^{*}$ es la medida de eficiencia orientada a insumos de la $D M U_{0}$. Debido a que no hay restricciones sobre los pesos $\lambda$, el modelo CCR toma como supuesto que las entidades exhiben rendimientos constantes a escala (CRS) supuesto que en el caso del sector bancario colombiano es muy fuerte debido a la diversidad de tamaños de los bancos. Por tanto, para permitir rendimientos variables a escala es necesario agregar una restricción de convexidad sobre los pesos $\lambda_{j}$. De esta manera al modelo CCR se le agrega la restricción:

$\sum_{j=1}^{n} \lambda_{j}=1$

$\mathrm{Al}$ incorporar la restricción (2) obtenemos el modelo BCC propuesto por Banker, Charnes y Cooper (1984), que permite retornos variables a escala:

$\theta^{*}=\min \theta-\varepsilon\left(\sum_{i}^{m} s_{i}^{-}+\sum_{i}^{m} s_{r}^{+}\right)$

$\sum_{j=1}^{n} x_{i j} \lambda_{j}+s_{i}^{-}=\theta x_{i 0}, i=1,2, \ldots, m ;$ 
$\sum_{j=1}^{n} y_{r j} \lambda_{j}-s_{r}^{+}=y_{r 0}, r=1,2, \ldots, s$

$\sum_{j=1}^{n} \lambda_{j}=1$

$\lambda, s_{r}^{+}, s_{i}^{-} \geq 0, \forall i, j, r$

Al ser una medida de eficiencia del grupo de comparación, en estos modelos el desempeño de una $D M U$ es totalmente eficiente, por definición, si y solo si $\theta^{*}=1$ y las holguras asociadas a sus restricciones son $s_{i}^{-*}=s_{r}^{+^{*}}=0$. No obstante, una firma puede ser débilmente eficiente al presentar $\theta^{*}=1$, pero a su vez las holguras asociadas a las restricciones son $s_{i}^{* *} \neq 0$ o $s_{r}^{*^{*}} \neq 0$. De igual forma se tiene que en el programa lineal (3) la cantidad $\varepsilon$ es un trascendental no arquimediano que indica que el problema debe resolverse en dos fases: en la primera se minimiza la medida radial de eficiencia $\theta$, y en la segunda se maximiza la suma de las holguras, manteniendo constante el valor de $\theta$ encontrado en la primera fase.

Así mismo se puede calcular la eficiencia orientada a productos transformando el modelo BCC anterior en:

$\varphi^{*}=\min \varphi-\varepsilon\left(\sum_{i}^{m} s_{i}^{-}+\sum_{i}^{m} s_{r}^{+}\right)$

$\sum_{j=1}^{n} x_{i j} \lambda_{j}+s_{i}^{-}=x_{i 0}, i=1,2, \ldots, m$.

$\sum_{j=1}^{n} y_{r j} \lambda_{j}-s_{r}^{+}=\varphi y_{r 0}, r=1,2, \ldots, s$.

$\sum_{j=1}^{n} \lambda_{j}=1$

$\lambda_{j}, s_{r}^{+}, s_{i}^{-} \geq 0, \forall i, j, r$

Una vez se obtienen los índices de eficiencia técnica se calcula la eficiencia de escala (SE) como el cociente entre la eficiencia obtenida del modelo CCR (retornos constantes a escala) y la eficiencia resultante del modelo BCC (retornos variables a escala):

$S E=\frac{C C R \theta^{*}}{B C C \theta^{*}}$

Al comparar los dos modelos se obtiene la escala en que las firmas operan, si $\sum_{j=1}^{n} \lambda_{j}^{*} \geq 1$ se sugiere retornos decrecientes a escala (DRS), en cambio, si $\sum_{j=1}^{n} \lambda_{j}^{*} \leq 1$ se encuentran retornos crecientes a escala (IRS) $\mathrm{y}$, finalmente, si $\sum_{j=1}^{n} \lambda_{j}^{*}=1$, se obtienen retornos constantes a escala (CRS). En este contexto las firmas se mueven en distintas escalas de producción. En algunas situaciones una firma puede lograr que un aumento en sus insumos se traduzca en un incremento proporcional de sus productos (CRS); algunas incrementan en una proporción mayor sus productos ante un aumento en sus insumos (IRS); mientras que otras veces este incremento puede ser menor en proporción al aumento en insumos. 
Para medir la eficiencia en costos se asume que las firmas minimizan costos, y se adopta un enfoque de insumos que se representa así: $\min c_{i 0} x_{i 0}$

$\sum_{j=1}^{n} x_{i j} \lambda_{j} \leq x_{i 0}, i=1,2, \ldots, m$.

$\sum_{j=1}^{n} y_{r j} \lambda_{j} \geq y_{r 0}, r=1,2, \ldots, s$.

$\sum_{j=1}^{n} \lambda_{j}=1$.

$\lambda_{j} \geq 0, j=1,2, \ldots, n$;

donde $c_{i 0}$ es el costo del insumo $i$ que enfrenta la firma analizada $\left(D M U_{0}\right)$. La medida de eficiencia en costos utiliza la relación:

$0 \leq \frac{\sum_{i=1}^{m} c_{i 0} x_{i}^{*}}{\sum_{i=1}^{m} c_{i 0} x_{i 0}} \leq 1$

donde $x_{i}^{*}$ representa los valores resultantes del modelo (6) y $x_{i 0}$ los valores observados para la $D M U_{0}$.

El índice Malmquist (IM) evalúa el cambio en la productividad de una firma entre dos periodos de tiempo y se define como el producto de los términos de cambio en la eficiencia técnica (Catch-up) y cambio tecnológico (Frontier-shift)

$I M=($ Catch up $) *($ Frontier shift $)$

E1 IM expresado en (8) se calcula como:

$M_{I}\left(X^{t+1}, Y^{t+1}, X^{t}, Y^{t}\right)=\frac{D_{I}^{t+1}\left(X^{t+1}, Y^{t+1}\right)}{D_{I}^{t}\left(X^{t}, Y^{t}\right)}\left[\left(\frac{D_{I}^{t}\left(X^{t+1}, Y^{t+1}\right)}{D_{I}^{t+1}\left(X^{t+1}, Y^{t+1}\right)}\right)\left(\frac{D_{I}^{t}\left(X^{t}, Y^{t}\right)}{D_{I}^{t+1}\left(X^{t}, Y^{t}\right)}\right)\right]^{1 / 2}$

Färe et al. (1994) definen el IM en (9) como la relación entre el cambio en eficiencia técnica al comparar la distancia de una firma a la frontera de eficiencia entre dos periodos de tiempo. Si el valor de esta razón es mayor a la unidad, la firma es más eficiente en el periodo $t+1$ frente al periodo $t$, lo que indica que se acerca más a la frontera en $t+1$. En el caso contrario, primer término de (9) inferior a 1 , se tiene que la firma se alejó de la frontera en $t+1$. El segundo componente mide el cambio técnico exhibido por la industria (conjunto de firmas evaluadas) al comparar la distancia entre las fronteras de eficiencia en $t$ y en $t+1$. Por tanto, si este segundo componente es superior a 1 , se dice que la industria presentó un cambio tecnológico positivo mejorando la eficiencia relativa de las firmas. El resultado de multiplicar los dos efectos es el IM. Si este índice es superior a la unidad la firma incrementó su productividad durante el periodo evaluado. Este incre- 
mento puede ser resultado de un aumento en la eficiencia técnica y/o un cambio tecnológico positivo que benefició toda la industria. Cabe mencionar que en presencia de retornos variables a escala (modelo $\mathrm{BCC}$ ) el cambio en eficiencia técnica (CE) o catch-up se divide en dos componentes: eficiencia técnica pura y eficiencia de escala:

$$
C E=\frac{D_{I}^{t+1}\left(X^{t+1}, Y^{t+1}\right)}{D_{I}^{t}\left(X^{t}, Y^{t}\right)}=\frac{D_{V R S}^{t+1}\left(X^{t+1}, Y^{t+1}\right)}{D_{V R S}^{t}\left(X^{t}, Y^{t}\right)} \times \frac{\frac{D_{C R S}^{t+1}\left(X^{t+1}, Y^{t+1}\right)}{D_{V S}^{t+1}\left(X^{t+1}, Y^{t+1}\right)}}{\frac{D_{C R S}^{t}\left(X^{t}, Y^{t}\right)}{D_{V R S}^{t}\left(X^{t}, Y^{t}\right)}}
$$

Para calcular IM con el enfoque DEA se asume que las funciones de distancia son recíprocas de las medidas de eficiencia técnica descritas en los modelos anteriores (Seiford y Thrall, 1990) ${ }^{4}$.

\section{DATOS Y DEFINICION DE VARIABLES}

Siguiendo estudios anteriores solo se seleccionaron bancos comerciales, debido a las diferencias en la actividad de cada tipo de entidad del sistema financiero. Estas diferencias pueden determinar funciones de tecnología y objetivo particulares para los distintos tipos de entidad, por lo cual un estudio agregado sobreestimaría las medidas de eficiencia (Hughes et al., 2001). Para el análisis se utilizaron datos trimestrales de una muestra de 23 bancos comerciales que operan en Colombia, la cual constituye un panel desbalanceado que cubre el periodo 2000-20095. Los bancos seleccionados representan el 87\% de los activos totales del sector. Los precios y niveles de insumos y productos se deflactaron a precios constantes de diciembre de 2009.

En el análisis de eficiencia técnica se propuso un modelo DEA de dos insumos y dos productos. Bajo el enfoque de intermediación, las entidades bancarias utilizan depósitos e incurren en ciertos niveles de costos asociados a su operación para generar inversiones y cartera de créditos (Hughes y Mester, 1993). Para el análisis de eficiencia en costos el modelo propuesto presenta dos productos y tres insumos con el respectivo costo asociado. El enfoque de intermediación para el modelo emplea los productos de la actividad bancaria como las inversiones realizadas por los bancos y los créditos ofrecidos. Para

\footnotetext{
${ }^{4}$ Para aplicaciones del índice de Malmquist a la industria bancaria, ver Wheelock y Wilson (1999) y Atkinson et al. (2003).

${ }^{5}$ Es un panel desbalanceado porque debido a las fusiones algunas entidades que figuraban al inicio del periodo desaparecen de la muestra porque fueron absorbidas o fusionadas. Las cifras se tomaron de las bases de datos del Banco de la República y la Superintendencia Financiera.
} 
generar estos productos los bancos utilizan tres insumos: capital laboral, financiero y físico. El precio del capital laboral $\left(C_{L}\right)$ es calculado como el cociente entre el gasto laboral y el número total de empleados, contratados y subcontratados. E1 precio del capital financiero $\left(C_{D}\right)$ es calculado como el cociente entre egresos financieros y el volumen total de depósitos; mientras que el precio del capital físico $\left(C_{k}\right)$ se obtiene dividiendo los gastos administrativos más las depreciaciones entre el activo físico total del banco. En el cuadro 1 se presentan las estadísticas descriptivas de las variables utilizadas en los modelos descritos ${ }^{6}$.

Cuadro 1

Estadísticas de las variables de los modelos DEA

(Miles de millones de pesos de 2009)

\begin{tabular}{lrrrr}
\hline Variable & \multicolumn{1}{c}{ Media } & \multicolumn{1}{c}{ Desv. Est. } & \multicolumn{1}{c}{ Min } & \multicolumn{1}{c}{ Max } \\
\hline Gastos de personal & $31.099,2$ & $33.796,7$ & 554,9 & $237.854,8$ \\
Egresos financieros & $69.400,8$ & $78.065,3$ & $3.743,0$ & $549.913,1$ \\
Gastos de administración & $52.049,1$ & $60.880,1$ & $1.939,7$ & $580.496,6$ \\
Depósitos & $3.885 .754,0$ & $4.660 .119,0$ & $146.005,1$ & $29.600 .000,0$ \\
Costo total & $152.549,1$ & $165.228,7$ & $11.791,4$ & $1.119 .157,0$ \\
Activo fijo & $93.036,7$ & $101.070,5$ & $5.359,1$ & $710.837,1$ \\
Activo total & $5.643 .130,0$ & $6.577 .083,0$ & $319.757,3$ & $41.700 .000,0$ \\
Inversiones & $1.357 .952,7$ & $1.472 .720,2$ & $32.466,7$ & $8.277 .268,0$ \\
Créditos & $3.305 .469,4$ & $4.195 .981,4$ & $132.508,6$ & $27.900 .000,0$ \\
\hline Fuente: SFC y Banco de la República, cálculos de los autores.
\end{tabular}

\section{RESULTADOS}

\section{Eficiencia técnica}

Este trabajo analiza la eficiencia reconociendo la naturaleza de las entidades bancarias en Colombia, cuyo nivel de bancarización es relativamente bajo y cuenta con un espacio suficiente para el crecimiento de las entidades. Este crecimiento solo se logra generando cada vez más productos en la operación bancaria. El modelo orientado a productos indica el nivel de producto (crédito e inversiones) que debería generar el banco, manteniendo sus costos e insumos. Bajo este supuesto todas las entidades bancarias emplean trabajo, activos físicos y niveles de depósitos a niveles acordes con su modelo de negocio y nicho de mercado. Por ello, el modelo se centra en la eficiencia técnica orientada a productos. Sin embargo, para observar

\footnotetext{
${ }^{6}$ La imposición de una periodicidad trimestral en el análisis en vez de mensual se debió a la imposibilidad de disponer datos mensuales de nómina para las entidades bancarias. Aun cuando para la mayoría de las variables había datos mensuales, una estimación mensual de la nómina a partir de la serie trimestral incurriría en supuestos muy fuertes dado el uso extensivo de subcontratación por parte de las entidades financieras y la movilidad del personal.
} 
la consistencia de las medidas de eficiencia, discutimos los resultados de ambas orientaciones ${ }^{7}$.

Cuadro 2

Medidas de eficiencia técnica y de escala promedio, 2000-2009 (Porcentajes)

\begin{tabular}{lcccccc}
\hline & \multicolumn{2}{c}{ Modelo orientado a productos } & \multicolumn{3}{c}{ Modelo orientado a insumos } \\
\cline { 2 - 7 } & $\begin{array}{c}\text { Eficiencia } \\
\text { técnica }\end{array}$ & $\begin{array}{c}\text { Eficiencia } \\
\text { técnica } \\
\text { Periodo }\end{array}$ & $\begin{array}{c}\text { Eficiencia } \\
\text { de escala }\end{array}$ & $\begin{array}{c}\text { Eficiencia } \\
\text { técnica } \\
\text { (BCC) }\end{array}$ & $\begin{array}{c}\text { Eficiencia } \\
\text { técnica } \\
\text { (CCR) }\end{array}$ & $\begin{array}{c}\text { Eficiencia } \\
\text { de escala }\end{array}$ \\
\hline 2000 & 73,1 & 66,9 & 91,9 & 72,1 & 66,9 & 93,5 \\
2001 & 72,4 & 67,6 & 93,6 & 71,4 & 67,6 & 95,1 \\
2002 & 74,7 & 71,8 & 96,2 & 74,1 & 71,8 & 97,0 \\
2003 & 77,5 & 75,0 & 96,8 & 77,0 & 75,0 & 97,6 \\
2004 & 77,1 & 74,4 & 96,5 & 77,0 & 74,4 & 97,0 \\
2005 & 78,9 & 75,7 & 96,1 & 78,6 & 75,7 & 96,6 \\
2006 & 85,0 & 81,5 & 95,9 & 85,1 & 81,5 & 96,0 \\
2007 & 87,3 & 84,1 & 96,3 & 87,1 & 84,1 & 96,4 \\
2008 & 85,4 & 79,4 & 93,0 & 85,3 & 79,4 & 93,2 \\
2009 & 86,5 & 81,4 & 94,2 & 86,3 & 81,4 & 94,7 \\
Total & 79,0 & 75,1 & 95,1 & 78,6 & 75,1 & 95,8 \\
\hline
\end{tabular}

Fuente: cálculos de los autores.

El cuadro 2 presenta los resultados del modelo de eficiencia técnica orientado a productos bajo el enfoque $\mathrm{BCC}$ propuesto en el programa lineal (4) y bajo el enfoque CCR, así como la medida de eficiencia de escala que resulta de comparar ambos enfoques. Los resultados muestran que, en promedio, la eficiencia orientada a productos para el sector bancario colombiano entre los años 2000 y 2009 fue de $79,0 \%$, presentando un promedio de eficiencia de escala del $95,1 \%$. Se observa también que bajo el modelo CCR el nivel de eficiencia técnica es inferior $(75,1 \%)$ debido a que éste asume retornos constantes a escala, mientras que el modelo BCC permite la presencia de retornos variables a escala entre las entidades. Bajo este enfoque, se destaca que en 2000 la banca colombiana presentaba un promedio de eficiencia técnica del 73\%, la cual aumentó gradualmente hasta alcanzar en 2007 un promedio de $87 \%$.

La crisis financiera global que se gestó a principios de 2008 parece haber afectado la eficiencia de la banca colombiana, que tuvo un retroceso de más del $2 \%$ frente al año anterior. Pese a ello, en la última década los bancos colombianos han logrado aumentar en $18,4 \%$ su

${ }^{7}$ La robustez de los resultados también se puede identificar mediante el uso de ventanas de tiempo. Evaluamos a las entidades bancarias en 33 ventanas diferentes de tiempo con dos años de duración cada una y encontramos que los niveles de eficiencia técnica fueron muy similares a los hallados con la aproximación global. Los resultados no se muestran por limitación de espacio, pero están disponible a solicitud. 
eficiencia que al cierre de 2009 fue de 86,5\%. Al observar los resultados del enfoque orientado a insumos, donde las entidades minimizan sus insumos dado el nivel de producto, se observa una tendencia de las tres medidas de eficiencia muy similar a la del enfoque de productos. La eficiencia técnica promedio orientada a insumos se estimó en 78,6\% en la última década, mientras que su nivel promedio de eficiencia de escala fue del 95,8\%. En el periodo de estudio hubo un crecimiento constante del indicador de eficiencia, con retrocesos en 2004 frente a 2003 y en 2008 frente a 2007.

Los índices de eficiencia del modelo BCC son consistentemente inferiores que los del enfoque de productos, lo cual era de esperar debido a que las entidades financieras buscan maximizar el producto. Asimismo, se destaca la consistencia bajo el modelo CCR, ya que al asumir retornos constantes a escala los resultados bajo ambas orientaciones deben ser los mismos.

La gráfica 1 muestra la evolución trimestral de las medidas de eficiencia técnica y de escala, y confirma el crecimiento permanente de ambas medidas de eficiencia técnica frente a la estabilidad de la medida de eficiencia de escala entre 2000 y el primer trimestres de 2007. A partir del segundo trimestre de 2007 se observa un deterioro en las medidas de eficiencia que se revierte en el segundo trimestre de 2009. Una tendencia similar se observa con el modelo orientado a insumos de la gráfica 2. La consistencia entre los resultados obtenidos en eficiencia técnica orientada a insumos y productos permite elegir uno de los dos enfoques para realizar los subsecuentes análisis en el estudio. De esta manera, acorde al supuesto de que los bancos operan a niveles de insumos adecuados para su particularidad de operación y nicho de mercado, el estudio continuará utilizando el enfoque orientado a productos, donde los bancos maximizan su producción, manteniendo constante su nivel de insumos.

Es importante mencionar que los resultados varían de forma significativa entre entidades. La gráfica 3 muestra el índice BBC orientado a productos para cada entidad evaluada durante el periodo de estudio. Se aprecia que tan solo diez entidades ( $43 \%$ de la muestra) registran niveles de eficiencia superiores al promedio del periodo (79\%). También se observa la presencia de un grupo de cuatro entidades que se ubican en los niveles de eficiencia técnica inferiores al 65\%. La discriminación de las medidas de eficiencia se complementó con un análisis de las entidades que fueron usadas en más ocasiones como referentes de comparación, lo cual permite identificar las entidades con las mejores prácticas del grupo. 
Gráfica 1

Eficiencia técnica y de escala para el modelo orientado a productos

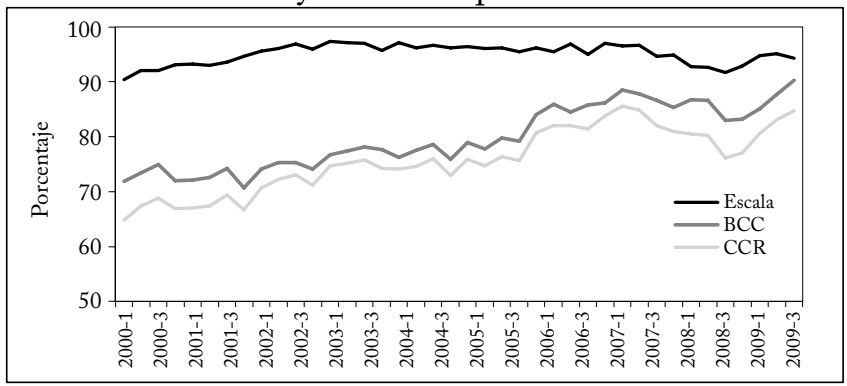

Fuente: cálculos de los autores.

\section{Gráfica 2}

Eficiencia técnica y de escala para el modelo orientado a insumos (Porcentaje)

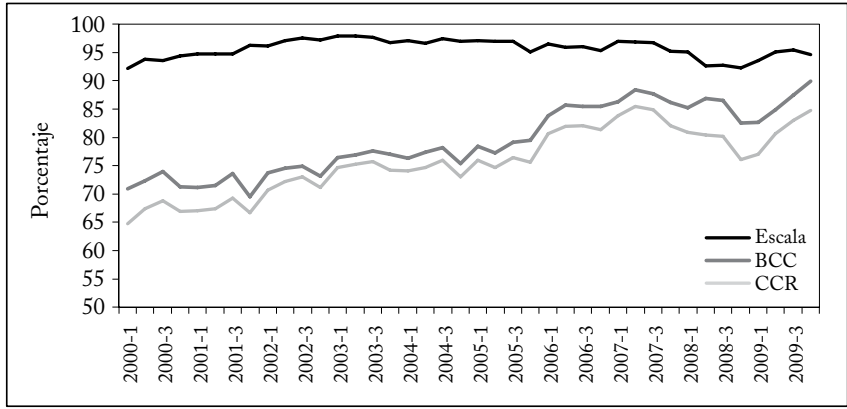

Fuente: cálculos de los autores.

\section{Gráfica 3}

Índice de eficiencia técnica (modelo BCC-productos), 2000-2009 (Porcentaje promedio por entidad)

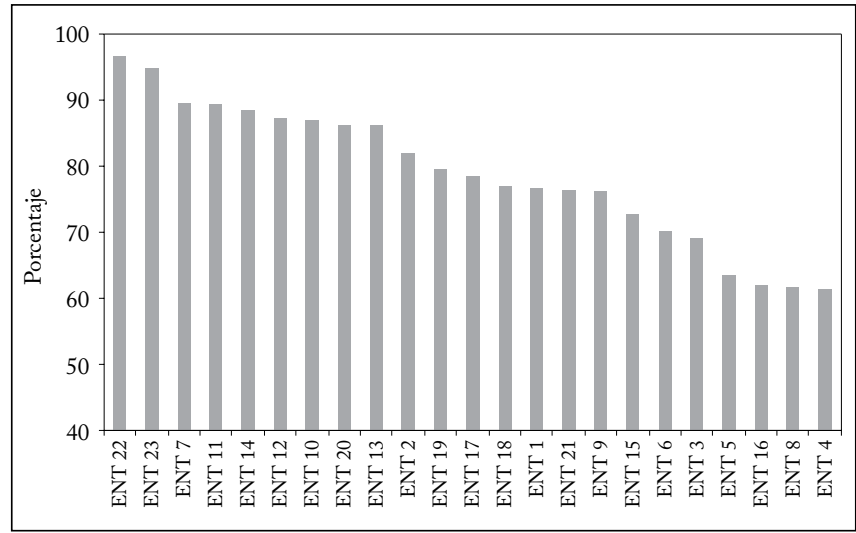

Fuente: cálculos de los autores.

Revista de Economía Institucional, vol. 20, n.o 38 , Primer semestre/2oi 8, pp. i57-i 83 


\section{Eficiencia en costos}

La eficiencia en costos se calculó mediante el programa lineal (6). Así, el modelo asume que una entidad utiliza personal, capital físico y depósitos a unos costos dados, para generar cartera de créditos e inversiones. Los resultados indican que en promedio la eficiencia en costos del sector durante el periodo se ubicó en $64,50 \%$, con una tendencia constante al alza, al pasar del 53,45\% en 2000 al 82,44\% al cierre de 2009. Al examinar los resultados trimestrales se observa que,

Gráfica 4

Eficiencia en costos trimestral promedio para el sector bancario (2000-2009) (Porcentaje)

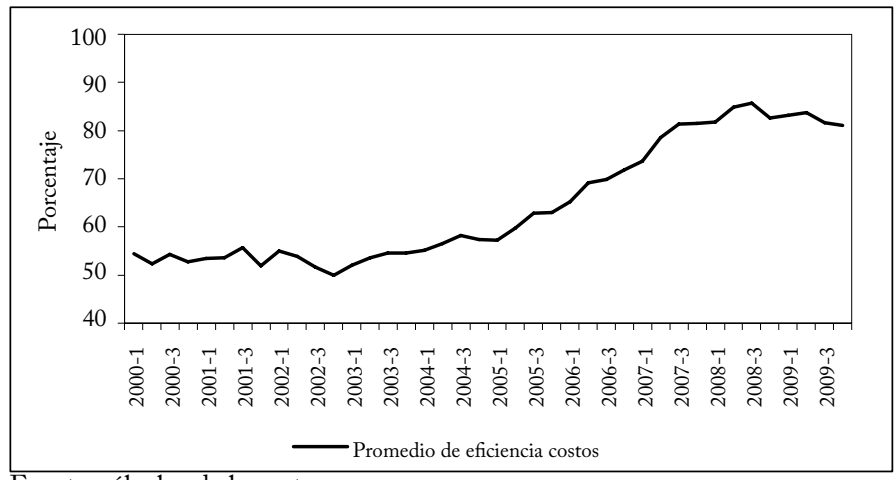

Fuente: cálculos de los autores.

\section{Gráfica 5}

Índice de eficiencia técnica en costos promedio 2000-2009 por entidad (Porcentaje)

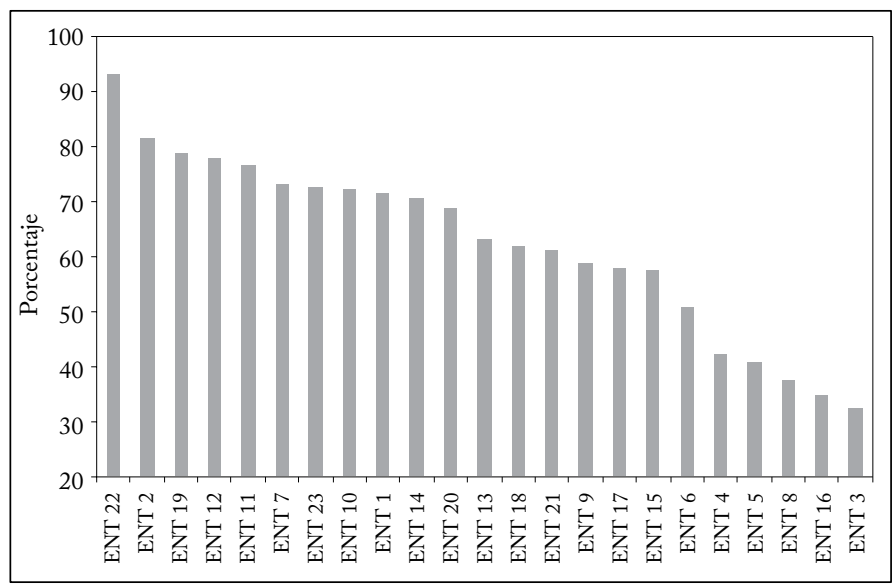

Fuente: cálculos de los autores. 
a diferencia de las medidas de eficiencia técnica, la eficiencia en costos no se recuperó después de la crisis de 2008 y continuó reduciéndose en 2009 (gráfica 4). Desde el enfoque DEA, este resultado sugiere que la estructura de costos es inflexible o que existe una respuesta tardía de las entidades en el ajuste de los costos de los insumos, lo cual no ha permitido que estos se adecúen a la caída en el nivel de producto a raíz del choque adverso de 2008. Cabe mencionar que esta característica de la industria bancaria ya se había evidenciado luego de la crisis financiera de 1999 (Clavijo et al., 2006).

$\mathrm{Al}$ interior de la industria se observan diferencias apreciables en términos de la eficiencia en costos con entidades que muestran niveles promedio desde 33\% hasta el 93\% durante el periodo (gráfica 5). Llama la atención que los niveles promedio de eficiencia en costos de las entidades son menores a los observados en la eficiencia, hecho que coincide con los hallazgos de Berger y Mester (2007) para los Estados Unidos y, a su vez, con los resultados de Estrada y Osorio (2004) para Colombia. Esta diferencia sugiere que existe independencia entre las medidas de eficiencia técnica orientada a productos (en beneficios) y la eficiencia en costos. Sin embargo, en la práctica estas menores eficiencias o mayores costos en la operación de la industria bancaria se traducen en mayores tarifas para los usuarios del sistema financiero ${ }^{8}$.

\section{ÍNDICE DE PRODUCTIVIDAD DE MALMQUIST}

El índice de productividad de Malmquist (MI) es el tercer enfoque utilizado para analizar la evolución de la eficiencia técnica de la banca colombiana, en el que se empleó el enfoque de la ecuación (9). Si bien el IM se ha empleado ampliamente en la industria bancaria no hay evidencia de esta medida de productividad en Colombia. E1 cálculo del IM para el periodo 2000-2009 indica que hubo un cambio favorable (ganancia) de productividad, del 3,9\% anual, en la industria bancaria. La mayor parte de este cambio $(74,4 \%)$ obedeció al cambio tecnológico (Frontier-shift), que mejoró en promedio un $2,9 \%$ anual. E1 25,6\% restante obedece a una mejora de la eficiencia técnica en cada entidad (Catch-up), que fue en promedio del $1 \%$ anual (cuadro 3 ).

8 A partir de la comparación entre la eficiencia en costos y beneficios, Turati (2003) sugiere que hay poder de mercado en el sector bancario europeo; específicamente afirma que los bancos ineficientes traducen mayores costos en mayores precios para el consumidor como una forma de mantener su beneficio. 
En todos los años, salvo en 2008, el cambio en productividad fue positivo (IM > 1), resultado que es consistente con lo observado en las medidas de eficiencia calculadas anteriormente. E1 mayor crecimiento se presentó en los periodos 2000-2001 (10,9\%) y 2005-2006 (10,7\%) seguido del periodo 2002-2004 (alrededor del 6\%). Este resultado también sugiere que la industria bancaria ha presentado un cambio tecnológico positivo que puede estar asociado a que las entidades se han beneficiado de forma similar de la tecnología disponible (innovación financiera) para aumentar su productividad con el tiempo.

Cuadro 3

Índice de Malmquist promedio anual de la industria bancaria, 2000-2009

\begin{tabular}{lccc}
\hline Años & $\begin{array}{c}\text { Índice de Malmquist } \\
(\mathrm{MI})\end{array}$ & $\begin{array}{c}\text { Cambio en eficiencia } \\
\text { técnica } \\
(\text { Catch-up })\end{array}$ & $\begin{array}{c}\text { Cambio } \\
\text { tecnológico } \\
\text { (Frontier-shift })\end{array}$ \\
\hline 2001 & 1,109 & 1,045 & 1,059 \\
2002 & 1,059 & 1,023 & 1,037 \\
2003 & 1,057 & 1,011 & 1,046 \\
2004 & 1,009 & 1,009 & 1,001 \\
2005 & 1,024 & 0,981 & 1,046 \\
2006 & 1,107 & 1,034 & 1,071 \\
2007 & 1,044 & 1,023 & 1,019 \\
2008 & 0,941 & 0,963 & 0,978 \\
2009 & 1,002 & 0,996 & 1,007 \\
$2000-2009$ & 1,039 & 1,010 & 1,029 \\
\hline
\end{tabular}

Fuente: cálculos de los autores.

En 2005, 2008 y 2009 el cambio en eficiencia técnica fue inferior a uno $(\mathrm{CE}<1)$ lo cual sugiere que en esos años las entidades (en promedio) se alejaron de su frontera de eficiencia (gráfica 6). Asimismo, se observa que la industria en su conjunto solo registró una caída (FS < 1) en 2008, hecho que confirma el efecto "sistémico" de la crisis sobre las entidades bancarias ${ }^{9}$. No obstante, se destaca la recuperación en las tres medidas a partir de 2009, hecho que también se ha observado en las anteriores medidas de eficiencia calculadas. Este resultado también fue hallado por Sarmiento y Galán (2017) y en particular lo asocian al efecto del riesgo sobre la eficiencia de la banca. El inherente riesgo de contagio por parte de las entidades que dependen de capital extranjero, acrecentó las restricciones de

9 Se denomina efecto sistémico porque su impacto afectó a todas las entidades evaluadas de forma similar. Un resultado similar fue encontrado por Decanay (2007) al evaluar el impacto de la crisis de finales de la década de 1990 sobre la industria bancaria en Filipinas. E1 impacto de los cambios regulatorios en Noruega también ha afectado la industria en su conjunto al ser evaluado por el IM (Berg et al., 1992). 
liquidez en el sistema, lo que propicia un ambiente de incertidumbre en la industria bancaria reduciendo su capacidad de incrementar el retorno de sus inversiones (Berger y Bowman, 2013). Ahn y Sarmiento (2017) encontraron que durante la crisis financiera de 2008, los bancos colombianos que fueron más expuestos a choques de liquidez redujeron la emisión de cartas de crédito que respaldan las importaciones realizadas por las firmas, lo cual contribuyó a la caída de las importaciones en ese periodo.

Gráfica 6

Índice de Malmquist, cambio en eficiencia técnica y cambio tecnológico (Promedio anual 2000-2009)

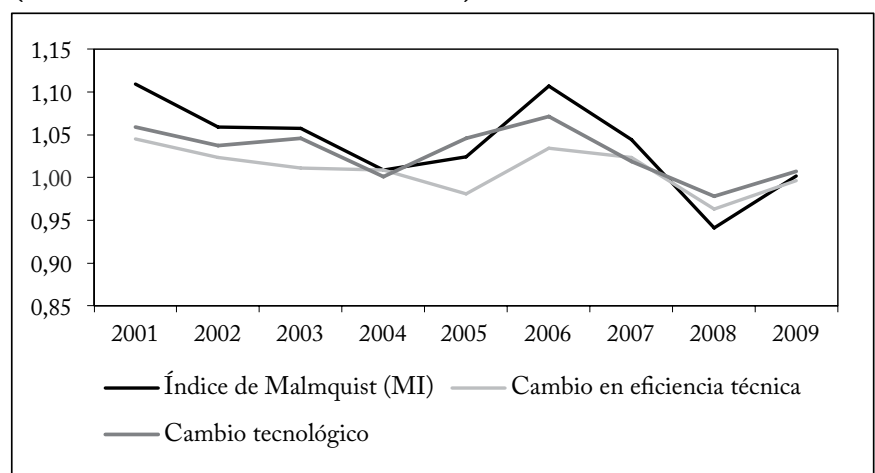

Fuente: cálculos de los autores.

\section{IMPACTO DE LAS F\&A EN LA EFICIENCIA DE LA INDUSTRIA BANCARIA}

En este apartado evaluamos el impacto de las F\&A sobre la eficiencia de las entidades absorbentes. Durante el periodo 2000-2009 se realizaron 21 procesos de F\&A en la industria bancaria de Colombia, siendo la década con mayores reorganizaciones en la historia reciente del país. Para evaluar el impacto de las F\&A se seleccionaron únicamente los procesos en donde tanto la entidad absorbente como la fusionada o adquirida fue un banco. Lo anterior debido a que las entidades son evaluadas bajo el enfoque de intermediación. Asimismo, se evaluaron aquellos procesos de fusión en donde se obtuvieron resultados de eficiencia para los 12 trimestres antes y después de la fusión, lo cual nos arroja una muestra final de siete F\&A para analizar ${ }^{10}$. Esto se hace

${ }^{10}$ Por limitaciones de espacio no se presentan los anexos con el detalle de los procesos de fusión y adquisición, los cuales están disponibles a solicitud del lector interesado. 
con el fin de incorporar los costos de ajuste que requiere el proceso de F\&A (Galán et al., 2015).

E1 análisis del impacto de las F\&A sobre la eficiencia de las entidades bancarias absorbentes se realizó mediante un proceso de tres etapas: la primera consiste en identificar el cambio estructural o periodo de fusión con base en los resultados de las eficiencias trimestrales, obtenidas por el modelo de eficiencia técnica BCC orientado a productos mediante la aproximación global. En la segunda fase se emplea el análisis por ventanas de tiempo para aislar los periodos post-fusión y pre-fusión de las series ${ }^{11}$. Así, utilizando los resultados obtenidos para cada entidad se conforman dos series con los promedios de eficiencia de los 12 trimestres anteriores y posteriores a la fusión. La tercera etapa consiste en aplicar la prueba no-paramétrica de comparación de medianas de Wilcoxon sobre las series obtenidas en la etapa 2, con el fin de identificar diferencias entre la eficiencia exhibida por la entidad antes y después de la fusión ${ }^{12}$.

En el cuadro 4 se presentan los resultados del test de Wilcoxon aplicado a las series de eficiencia técnica ex-ante y ex-post de las entidades absorbentes en los siete procesos de F\&A evaluados. Las pruebas de hipótesis indican que existe evidencia estadística para rechazar la hipótesis nula de que las medianas son iguales en ambas series y a su vez, se acepta la hipótesis alternativa en la cual la eficiencia después de la fusión es significativamente mayor a la presentada por la entidad antes de ella. Lo anterior sugiere que existe evidencia a favor del incremento en la eficiencia de las entidades absorbentes luego de realizar un proceso de F\&A, debido a que estas presentaron niveles sistemáticamente superiores de eficiencia después del proceso de fusión.

En la gráfica 7 se presentan los índices de eficiencia técnica obtenidos para las entidades absorbentes en tres F\&A seleccionadas. Se observa que en la F\&A 3 (panel a) la principal caída en los indicadores de eficiencia se presenta en el tercer trimestre de 2005 en el índice CCR, periodo que coincide con la fusión que efectúo esta entidad. Asimismo, se aprecia una ganancia importante en la eficiencia BCC durante ese mismo periodo, que se mantiene luego de la fusión. Nótese que en la prueba de Wilcoxon no se rechaza la

${ }^{11} \mathrm{E} 1$ trimestre en donde ocurre la fusión no es incluido en ninguna de las dos series pues está afectado por las dificultades administrativas que enfrentan las entidades en la reorganización del personal y activos, entre otros aspectos, los cuales se ven reflejados en la unión de cuentas y balances.

${ }^{12}$ La prueba no-paramétrico de comparación de medianas de Wilcoxon (1945) ofrece mejores resultados en muestras cortas y asimétricas que su homólogo paramétrico t-student.

Revista de Economía Institucional, vol. 20, n.o 38, primer semestre/20i8, pp. I57-i 83 
Cuadro 4

Prueba de medianas de Wilcoxon

Series pre-fusión y post-fusión de las entidades absorbentes en las F\&A

\begin{tabular}{|c|c|c|c|c|c|c|c|}
\hline Hipótesis $^{1}$ & F\&A 1 & F\&A 2 & F\&A 3 & F\&A 4 & F\&A 5 & F\&A 6 & F\&A 7 \\
\hline $\begin{array}{l}\text { Ho: } \text { Mediana }=0 \\
\text { Ha: } \text { Mediana }>0\end{array}$ & 0,997 & 0,971 & 0,981 & 0,999 & 0,999 & 0,999 & 0,994 \\
\hline $\begin{array}{l}\text { Ho: } \text { Mediana }=0 \\
\text { Ha: } \text { Mediana }<0\end{array}$ & $0,035^{\text {*** }}$ & $0,068^{*}$ & $0,073^{*}$ & $0,003^{\text {**** }}$ & $0,003^{* * * *}$ & $0,003^{* * * *}$ & 0,032 \\
\hline $\begin{array}{l}\text { Ho: Mediana }=0 \\
\text { Ha: Mediana } \neq 0\end{array}$ & $0,072^{*}$ & $0,092^{*}$ & 0,146 & $0,006^{* * * *}$ & $0,008^{* * * * *}$ & $0,006^{* * * *}$ & $0,065^{*}$ \\
\hline
\end{tabular}

hipótesis nula de diferencia de medianas ex-ante y ex-post al 14\%, nivel estadísticamente bajo lo que indicaría que no se presenta una diferencia significativa en el nivel de eficiencia de la entidad. Sin embargo, existe evidencia, al 93\% de confianza, de que luego de la fusión la eficiencia se incrementó de forma importante, lo cual también se confirma en la gráfica. En el caso de la F\&A 4 el periodo de la fusión (segundo trimestre de 2006) coincide con una caída en ambos índices de eficiencia técnica en una magnitud superior al 30\% (panel b). Una vez efectuado el proceso de fusión la entidad exhibe un rápido incremento en las medidas de eficiencia, con niveles comparativamente mayores a los registrados antes de la fusión. Una tendencia similar se observa en la F\&A 7 realizada en el cuarto trimestre de 2006 (panel c). A diferencia de las anteriores fusiones, las medidas de eficiencia de esta entidad se incrementaron en más de 20 puntos porcentuales después de la fusión. De esta forma, la evidencia está en línea con los hallazgos para Colombia (Galán et al., 2015) y la evidencia internacional sobre las ganancias en eficiencia después de los procesos de F\&A (Davis, 2007; DeYoung et al., 2009).

\section{CONSIDERACIONES FINALES}

En este artículo se empleó el método no paramétrico DEA para evaluar la eficiencia de la industria bancaria en Colombia entre 2000 y 2009, utilizando el enfoque de intermediación financiera. Los resultados sugieren que la industria bancaria en Colombia ha exhibido un incremento en sus niveles de eficiencia, aunque registró una fuerte caída en 2008. La caída del consumo externo y la inversión afectaron 


\section{Gráfica 7}

Medidas de eficiencia, entidades absorbentes seleccionadas en las F\&A (Modelo orientado a productos bajo la aproximación global)

Panel a. F\&A 3 (III-2005)

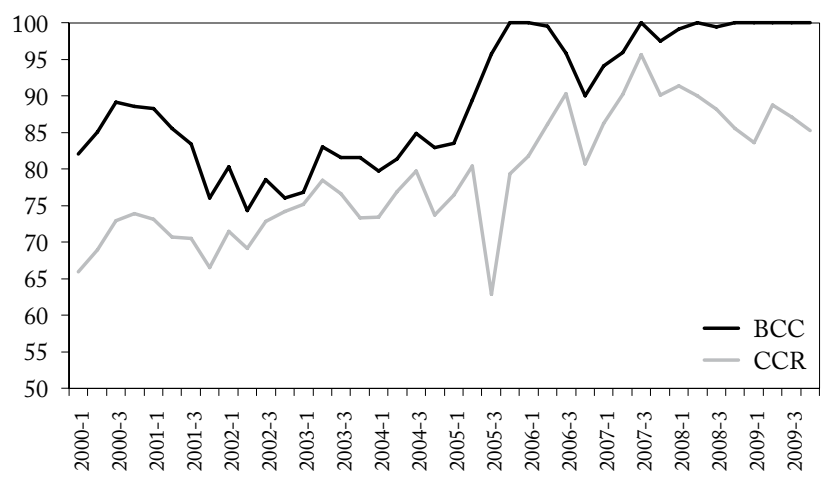

Panel b. F\&A 4 (II-2006)

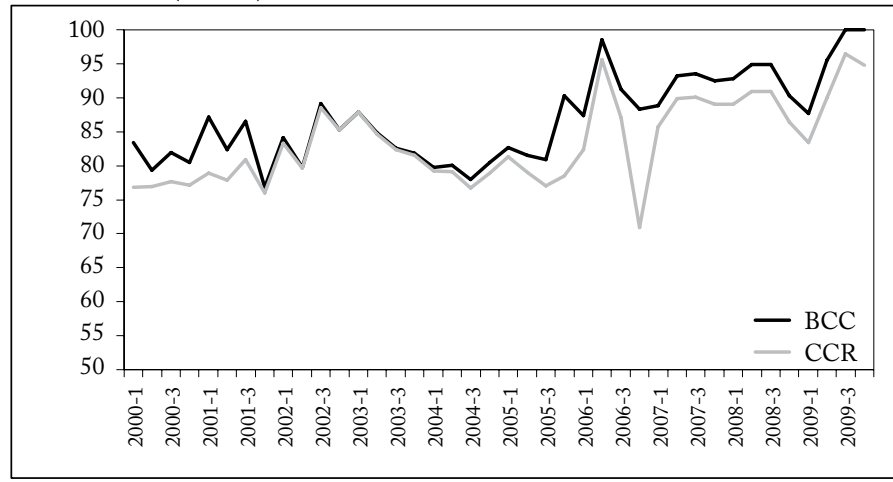

Panel c. F\&A 7 (IV-2006)

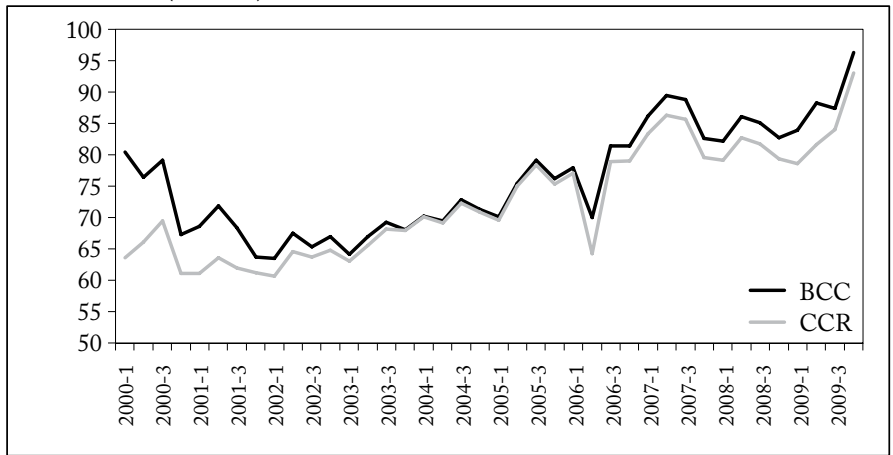

Fuente: cálculos de los autores. 
directamente la habilidad de los bancos para incrementar el crédito, lo cual asociado a un nivel relativamente estable de insumos, se tradujo en una reducción generalizada de los índices de eficiencia, siendo la eficiencia en costos la más afectada. Este resultado indica que existen costos de ajuste importantes en la banca, característica que ya había sido observada en otros estudios para Colombia luego de la crisis financiera de finales de los noventa (Clavijo et al., 2006).

El cálculo del índice de Malmquist, que por primera vez se estima para la banca en Colombia, mostró que la productividad en la industria bancaria amentó en promedio 3,9\% durante el periodo, crecimiento que se explica en mayor medida por el cambio tecnológico experimentado en la industria (77\%) y, en menor grado, por el aumento de la eficiencia técnica de las entidades (23\%). Asimismo, se observa que la industria en su conjunto solo registró una caída en este índice en 2008, hecho que confirma el efecto sistémico de la crisis financiera global que afectó a todas las entidades bancarias en Colombia. No obstante, se destaca la recuperación del índice y sus componentes a partir de 2009.

Las F\&A de los bancos han contribuido de manera importante al aumento de la eficiencia bancaria, pues las entidades absorbentes buscan economías de escala que se traducen en menores costos de operación y ganancia de mercado. Para futuros estudios se sugiere estudiar la sensibilidad de las medidas de eficiencia ante choques locales y externos. También es necesario profundizar el estudio de los efectos de la regulación bancaria en la eficiencia de la banca, pues el incremento de los requerimientos de capital exigidos por Basilea III exigirá mayor eficiencia de la industria bancaria para evitar el aumento de los costos de intermediación, que se traduciría en mayores costos para la economía.

\section{REFERENCIAS BIBLIOGRÁFICAS}

Ahn, J. y Sarmiento, M. (2017). Estimating the direct impact of bank liquidity shocks on the real economy: Evidence from letter-of-credit import transactions in Colombia. Mimeo, Washington DC: FMI.

Aigner, D., Lovell, C. y P. Schmidt. (1977). Formulation and estimation of stochastic frontier production function models. Journal of Econometrics, 1(6), 21-37.

Alam, I. M. (2001). A nonparametric approach for assessing productivity dynamics of large U.S. banks. Journal of Money, Credit and Banking, 33(1), 121-139.

Allen, J., Engert, W. y Liu, Y. (2006). Are Canadian banks efficient? A Canada-U.S. comparison. Bank of Canada working paper 2006-33. 
Almanza, C. (2012). Eficiencia en costos de la banca en Colombia, 1999-2007: una aproximación no paramétrica. Innovar, 22(44), 67-78.

Altunbas, Y., Gardener, E., Molyneux, P. et al. (2001). Efficiency in European banking. European Economic Review, 45, 1931-1955.

Atkinson, S., Cornwell, C. y Honerkamp, O. (2003). Measuring and decomposing productivity change: Stochastic distance function estimation versus data envelopment analysis. Journal of Business and Economic Statistics, 21(2), 284-294.

Banker, R., Charnes, A. y Cooper, W. (1984). Some models for estimating technical and scale inefficiencies in data envelopment analysis. Management Science, 9(30), 1078-1092.

Barajas, A., Steiner, R. y Salazar, A. (2002). The impact of liberalization and foreign direct investment in Colombia's financial sector. Journal of Development Economics, 23, 157-196.

Battese, G. y Coelli, T. (1995). A model for technical inefficiency effects in stochastic frontier production function for panel data. Empirical Economic, 20(2), 325-332.

Berg, S., Førsund, F. y Jansen, E. (1992). Malmquist indices of productivity growth during the deregulation of Norwegian banking 1980-89. Scandinavian Journal of Economics, 94 (supplement), 211-228.

Berger, A. y Bowman, C. (2013). How does capital affect bank performance during financial crises? Journal of Financial Economics, 109(1), 146-176.

Berger, A. y Humphrey, D. (1997). Efficiency of financial institutions: International survey and directions for future research. European Journal of Operational Research, 98(2), 175-212.

Berrío, D. y Muñoz, S. (2005). Análisis de la eficiencia relativa del sistema bancario en Colombia en el periodo 1993-2003 y propuesta estratégica de fortalecimiento. Pensamiento y Gestión, 18, 1-36.

Carletti, E., Hartmann, P. Y Spagn, G. (2007). Bank mergers, competition, and liquidity. Journal of Money Credit E Banking, 39(5), 1067-1105.

Casu, B., Girardone, C. y Molyneux, P. (2004). Productivity change in European Banking: A comparison of parametric and non-parametric approaches. Journal of Banking E Finance, 28, 2521-2540.

Caves, R., Christensen, L. y Diewert, W. (1982). The economic theory of index numbers and the measurement of input, output and productivity. Econometrica, 50, 1393-1414.

Cepeda, A., Sarmiento, M. y Mutis, H. (2010). Eficiencia del sector bancario en Colombia 2000-2009: una medición a partir de frontera estocástica. Mimeo, presentado en el XX Simposio Nacional de Estadística de la Universidad Nacional de Colombia, Santa Marta.

Chansarn. S. (2008). The relative efficiency of commercial banks in Thailand: DEA Approach. International Research Journal of Finance and Economics, 18, 53-68.

Charnes, A., Cooper, W. y Rhodes, E. (1978). Measuring the efficiency of decision making units. European Journal of Operational Research, $2,429-444$. 
Claus, I., Jacobsen, V y Jera, B. (2004). Financial systems and economic growth: An evaluation framework for policy. Wellington, New Zealand Treasury working paper 04/17.

Clavijo, S., Rojas, C., Salamanca, C. et al. (2006). Mergers and acquisitions in the Colombian Financial Sector: Impact on Efficiency, 1990-2005. Bogotá: ANIF.

Coelli, T., Prasada, D. y Battese, G. (1998). An introduction to efficiency and productivity analysis. Boston: Kluwer Academic Publisher.

Cooper, W., Seiford, L., y Zhu, J. (2004). Handbook on data envelopment analysis. Boston: Kluwer Academic Publishers.

Davis, K. (2007). Banking concentration, financial stability and public policy. Mimeo, Reserve Bank of Australia Conference.

Decanay, S. J. (2007). Malmquist index and technical efficiency of Philippine commercial banks in the post-Asian financial crisis period. Philippine Management Review, 14, 93-114.

DeYoung, D., Evanoff, D. y Molyneux, P. (2009). Mergers and acquisitions of financial institutions: A review of the post-2000 literature. Journal of Financial Services Research, 36(2-3), 87-110.

Dias, D., Huang, Y., Rey, H. et al. (2017). International channels of monetary policy: Evidence of financial and real effects on emerging markets. Mimeo, Bogotá: Banco de la República.

Dolar, V. y Meh, C. (2002). Financial structure and economic growth: $A$ non-technical survey. Bank of Canada, working paper 2002-24.

Estrada, D., y Osorio, P. (2004). Efectos del capital financiero en la eficiencia del sistema bancario colombiano. Ensayos sobre Politica Económica, 47. Bogotá: Banco de la República.

Färe, R., Grifell-T., E., Grosskopf, S. et al. (1997). Biased technical change and the Malmquist productivity index, Scandinavian Journal of Economics, 99(1), 119-127.

Färe, R., Grosskopf, S. Norris, S. et al. (1994). Productivity growth, technical progress, and efficiency changes in industrialized countries. American Economic Review, 84, 66-83.

Farrell, M. J. (1957). The measurement of productive efficiency. Journal of Royal Statical Society, 120, 253-290.

Fiorentino, E., Karmann, A. y Koetter, M. (2006). The cost efficiency of German banks: A comparison of SFA and DEA. Discussion Paper Series 2: Banking and Financial Studies, 10, Deutsche Bundesbank.

Galán, J., Veiga, H. y Wiper, M. (2015). Dynamic effects in inefficiency: Evidence from the Colombian banking sector. European Journal of Operational Research, 240(2), 562-571.

Galán, J. E. y Sarmiento, M. (2008). Banknote printing at modern central banking: Trends, costs and efficiency. Money Affairs, 21(2), 217-262.

Gómez, E., Lizarazo, A., Mendoza, J. y Murcia, A. (2016). Evaluating the impact of macroprudential policies in Colombia. Mimeo, Bogotá: Banco de la República.

Herrera, S. y Pang, G. (2005). How efficient is public spending in education? Ensayos sobre Politica Económica, 51, Bogotá: Banco de la República, 136-201. 
Hughes, J., Mester, L. y Moon, C. (2001). Are scale economies in banking elusive or illusive? Evidence obtained by incorporating capital structure and risk-taking into models of bank production. Journal of Banking Eं Finance, 25(12), 2169-2208.

Hughes, J. y Mester, L. (1993). A quality and risk-adjusted cost function for banks: Evidence on the too-big-to-fail doctrine. Journal of Productivity Analysis, 4(3), 293-315.

Hughes, J., Land, W., Mester, L. et al. (1996). Efficient banking under interstate branching. Journal of Money, Credit, and Banking, 28(4), 1045-1071.

Jemric, I. y Vujcic, B. (2002). Efficiency of banks in Croatia: A DEA approach. Comparative Economic Studies, 44(2-3), 169-193.

Jonas, M. y King, S. (2008). Bank efficiency and the effectiveness of monetary policy. Contemporary Economic Policy, 26, 579-589.

Koetter, M. (2008). An assessment of bank merger success in Germany. German Economic Review, 9(2), 232-264.

Kumbhakar, S. y Lovell, C. (2000). Stochastic frontier analysis. Cambridge, Mass.: Cambridge University Press.

León, C., Machado, C., Cepeda, F. et al. (2012). Systemic risk in large value payment systems in Colombia: A network topology and payments simulation approach. En M. Hellqvist y T. Laine (eds.), Diagnostics for the financial markets, computational studies of payment system (pp. 267-313). Helsinki: Bank of Finland.

Pirateque, J., Piñeros, J. y Mondragón, L. (2013). Eficiencia de los establecimientos bancarios (EB): una aproximación mediante modelos DEA. Borradores de Economía 798. Bogotá: Banco de la República.

Resti, A. (1997). Evaluating the cost efficiency of the Italian banking system: What can be learned from the joint application of parametric and nonparametric techniques. Journal of Banking E Finance, 21(2), 221-250.

Sarmiento, M. (2006). ¿Cómo medir la eficiencia del sector público? Lecciones para Colombia a partir de los recientes enfoques de frontera eficiente. Mimeo, Seminario Técnico del Departamento Nacional de Planeación. Bogotá: DNP.

Sarmiento, M. y Galán, J. (2017). The influence of risk-taking on bank efficiency: Evidence from Colombia. Emerging Markets Review, 32, 52-73.

Seiford, L. y Thrall, R. (1990). Recent developments in DEA: The mathematical approach to frontier analysis. Journal of Econometrics, 46(1-2), 7-38.

Staub, R., Da Silva, G. y Tabak, B. (2010). Evolution of bank efficiency in Brazil: A DEA approach. European Journal of Operational Research, 202(1), 204-213.

Turati, G. (2003). Cost efficiency and profitability in European commercial banking: Implications for antitrust analysis. Tesis de doctorado, Universidad de Turín.

Wang, H., y Schmidt, P. (2002). One step and two step estimation of the effects of exogenous variables on technical efficiency levels. Journal of Productivity Analysis, 18(2), 129-144. 
Weill, L. (2004). Measuring cost efficiency in European banking: A comparison of frontier techniques. Journal of Productivity Analysis, 21(2), 133-152.

Wheelock, D. y Wilson, P. (2003). Robust non-parametric estimation of efficiency and technical change in U.S commercial banking. Federal Reserve Bank of St. Louis working paper.

Wheelock, D. y Wilson, P. (1999). Technical progress, inefficiency and productivity change in U.S. banking, 1984-1993. Journal of Money, Credit and Banking, 31(2), 213-234.

Wilcoxon, F. (1945). Individual comparisons by ranking methods. Biometrics Bulletin, 1(6), 80-83. 